\title{
Model-Based Analysis of Local Shape for Lesion Detection in CT Scans
}

\author{
Paulo R.S. Mendonça ${ }^{1}$, Rahul Bhotika ${ }^{1}$, Saad A. Sirohey ${ }^{2}$, Wesley D. Turner ${ }^{1}$, \\ James V. Miller ${ }^{1}$, and Ricardo S. Avila ${ }^{3}$ \\ ${ }^{1}$ GE Global Research, One Research Circle, Niskayuna, NY 12309, USA \\ \{mendonca, bhotika, turner, millerjv\}@research.ge.com \\ ${ }^{2}$ GE Healthcare, 3200 N Grandview Blvd, Waukesha, WI, 53188, USA \\ saad.sirohey@ge.com \\ 3 Kitware Inc., 28 Corporate Drive, Suite 204, Clifton Park, NY 12065, USA \\ rick.avila@kitware.com
}

\begin{abstract}
Thin-slice computer tomography provides high-resolution images that facilitate the diagnosis of early-stage lung cancer. However, the sheer size of the CT volumes introduces variability in radiological readings, driving the need for automated detection systems. The main contribution of this paper is a technique for combining geometric and intensity models with the analysis of local curvature for detecting pulmonary lesions in CT. The local shape at each voxel is represented via the principal curvatures of its associated isosurface without explicitly extracting the isosurface. The comparison of these curvatures to values derived from analytical shape models is then used to label the voxel as belonging to particular anatomical structures, e.g., nodules or vessels. The algorithm was evaluated on 242 CT exams with expert-determined ground truth. The performance of the algorithm is quantified by free-response receiver-operator characteristic curves, as well as by its potential for improvement in radiologist sensitivity.
\end{abstract}

\section{Introduction}

State-of-the-art computer tomography (CT) scanners, with spatial resolutions of less than a millimeter, routinely provide images of smaller and smaller nodules, characteristic of early-stage lung cancer [1]. However, these gains in spatial resolution have led to an explosion in the sizes of the image volumes that a radiologist has to review, resulting in significant variability in radiological readings [2]. Figure 1] shows examples of nodules detected by two expert radiologists but missed by a third expert.

Computer-aided detection (CAD) systems have been developed to aid radiologists in reading CT exams. Brown et al [3] use simple shape- and intensity-based features of segmented regions with a fuzzy classifier. The data consisted only of selected $2 \mathrm{~cm}$ thick cross-sections of the lung, favoring the 3D segmentation technique upon which the algorithm is dependent and avoiding the difficult apex region. McCulloch et al [4] obtained encouraging results with a Bayesian classifier - 70\% sensitivity at 8 false positives per case operating on noisy low-dose screening data. Their method uses $2 \mathrm{D}$ segmentation to generate candidates and the number of false positives grows with the use of thinner slices in CT. Paik et al [5] use a geometric model and learn parameters from training data. They report results on only $8 \mathrm{CT}$ volumes and on nodules with 


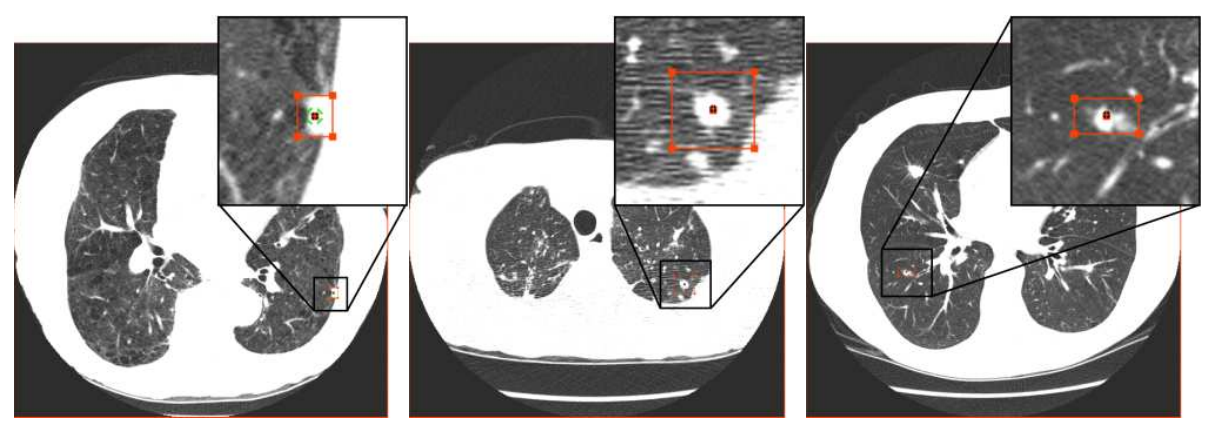

Fig. 1. Three examples of structures missed by an expert radiologist but independently classified as nodules by two other experts. These abnormalities were successfully detected by the algorithm proposed here.

diameters above $6 \mathrm{~mm}$, thus no solid conclusions can be drawn. Farag et al [6] report numbers as high as $82.3 \%$ sensitivity with $9.2 \%$ false positive rate. However, the majority of their nodules are calcified and therefore clinically irrelevant [7] and over $80 \%$ of their ground truth consists of nodules above $12 \mathrm{~mm}$ which are easy to detect. Others have used local structure information alone to discriminate between particular shapes [8, 9, 10]. However, in all of these methods the thresholds for classification are either set empirically or learned from data.

This paper introduces a technique for the shape analysis of 3D images and demonstrates its application to the detection of lung nodules in CT exams. The method is based on a fully $3 \mathrm{D}$ algorithm that does not depend on segmenting or grouping the relevant anatomical structures or any edge detection method. The proposed algorithm combines geometric and intensity models with the eigenanalysis of the curvature tensor in order to identify pulmonary nodules in CT. All parameters and thresholds are derived from these models, eliminating the need for training data. Furthermore, no model fitting or optimization is performed. All operations are local and therefore the proposed method can be interpreted as a filter for highlighting nodule-like structures in the image.

\section{Differential Operators on Volume Images}

A volume image $I$ is defined as a twice-differentiable $\left(\mathcal{C}^{2}\right)$ mapping from a compact domain $\mathcal{V} \subset \mathbb{R}^{3}$ into $\mathbb{R}$. For any given $k$, the equation

$$
I(\mathbf{x})=k
$$

defines an isosurface $M_{k} \subset \mathcal{V}$ at the points $\mathbf{x}$ satisfying (1) and $\nabla I(\mathbf{x}) \neq 0$ [11]. A common descriptor for the local structure of an image $I$ is its Hessian $\mathbf{H}$ [8]. Although the eigenvalues of $\mathbf{H}$ provide an intuitive measure of local structure, they do not capture the true underlying shape, which is more accurately described by the curvature of the isosurfaces defined by (11). For example, consider an isotropic Gaussian intensity profile (see Fig. 22). Hessian-based shape measures [8] would incorrectly signal the presence of a cylinder at the inflection points of the profile, while the principal curvatures would correctly flag the entire structure as spherical. 


\subsection{The Curvature Tensor}

It can be shown that, at the point $\mathbf{x}$, the principal directions of the isosurface given by (11) can be obtained directly from the implicit function by solving the eigenproblem

$$
\begin{aligned}
& \min _{\hat{\mathbf{v}}} / \max _{\hat{\mathbf{v}}} \frac{-\hat{\mathbf{v}}^{\mathrm{T}} \mathbf{N}^{\mathrm{T}} \mathbf{H N} \hat{\mathbf{v}}}{\|\nabla I\|}, \\
& \text { subject to }\|\hat{\mathbf{v}}\|=1,
\end{aligned}
$$

where $\mathbf{N}$ is the $3 \times 2$ matrix of the null space of $\nabla I$. The principal directions $\mathbf{v}_{1}$ and $\mathbf{v}_{2}$ are given by $\mathbf{v}_{1,2}=\mathbf{N} \hat{\mathbf{v}}_{1,2}$, where $\hat{\mathbf{v}}_{1,2}$ are the solutions of (2), and the corresponding principal curvatures $\kappa_{1}$ and $\kappa_{2}$ are the eigenvalues of the $2 \times 2$ matrix $-\mathbf{N}^{\mathrm{T}} \mathbf{H N} /\|\nabla I\|$, with $\kappa_{1} \leq \kappa_{2}$. The matrix $\mathbf{C}=-\mathbf{N}^{\mathrm{T}} \mathbf{H N} /\|\nabla I\|$ is herein defined as the curvature tensor of the volume image.

Yoshida et al [9] present a method to compute $\kappa_{1}$ and $\kappa_{2}$ for an implicit surface. Their technique also estimates curvatures directly from an implicit function. However, it requires the isosurfaces to be amenable to a local parameterization via Monge patches, which cannot be achieved everywhere on the surface [12]. The solution from [2] circumvents this problem and also avoids the rotation step required by Vos et al [10].

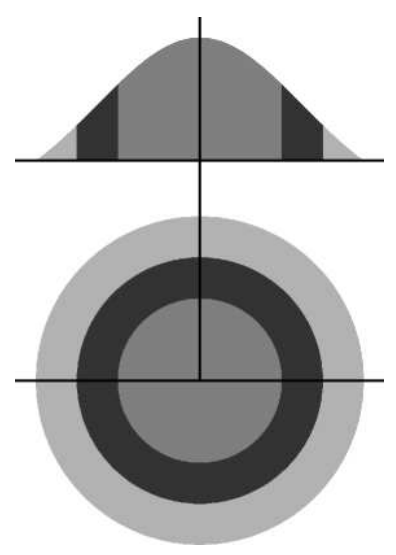

Fig. 2. Cross-section and top view of Hessian responses on a $2 \mathrm{D}$ Gaussian profile. The Hessian response is spherical in the innermost circle, cylindrical in the dark band containing the inflection point, and void in the outer band.

\subsection{Response of the Curvature Tensor}

At points $\mathbf{x}$ for which the isosurface defined by (1) is well-approximated by a spherical patch with surface normal pointing outward, $\kappa_{1}$ and $\kappa_{2}$ satisfy $\kappa_{1}>0$ and $\kappa_{2}>0$. For cylindrical patches with an outward normal, $\kappa_{1} \approx 0$ and $\kappa_{2}>0$. Finally, saddle points have $\kappa_{1}<0$ and $\kappa_{2}>0$. This suggests a methodology to discriminate between spherical and cylindrical regions in volume images, such as nodules and vessels in CT scans of the lung. The sphericalness and cylindricalness measures $b(\mathbf{x})$ and $c(\mathbf{x})$ for the curvature tensor at the isosurface intersecting the point $\mathbf{x}$ are defined as

$$
b(\mathbf{x})=\left\{\begin{array}{ll}
\frac{\kappa_{1}}{\kappa_{2}} & \text { if } \kappa_{1}, \kappa_{2}>0, \\
0 & \text { otherwise, }
\end{array} \text { and } c(\mathbf{x})= \begin{cases}1-\frac{\kappa_{1}}{\kappa_{2}} & \text { if } \kappa_{2}>0, \\
0 & \text { otherwise }\end{cases}\right.
$$

\section{Anatomical Modeling}

The eigenvalues of the curvature tensor can be used to quantify the sphericalness or cylindricalness of a voxel in a CT volume by thresholding the values attained by (3) at that voxel. An important contribution of this work is the use of a model-based approach to set these thresholds from generative models for the relevant anatomical structures. 


\subsection{Anatomical Modeling of Local Shape}

The eigenvalues $\kappa_{1}$ and $\kappa_{2}$ of the curvature tensor are independent of any transformation $I^{\prime}(\mathbf{x})=a I(\mathbf{x})+b$ applied to the image volume $I(\mathbf{x})$. In fact, $\kappa_{1}$ and $\kappa_{2}$ at a voxel $\mathbf{x}$ depend only on the shape of the associated isosurface, not on its isovalue. Therefore one only needs to take into account the local shapes of the relevant anatomical structures, not the exact form of their intensity profiles.

Radiologists routinely model pulmonary nodules as spherical objects when attempting to measure quantities such as volume and growth rate [13]. In this work, the local shapes of isosurfaces of relevant 3D anatomical structures - nodules and vessels are approximated using ellipsoidal and toroidal surface patches.

Ellipsoidal model. Under the appropriate curvature-preserving mapping the implicit equation of an ellipsoid $\mathcal{E}$ is

$$
\frac{x^{2}}{a^{2}}+\frac{y^{2}}{b^{2}}+\frac{z^{2}}{c^{2}}=1,
$$

where $a \leq b \leq c$ are the lengths of the ellipsoid's semi-axes, as shown in Fig. 3 a). The principal curvatures $\kappa_{1}^{\mathcal{E}}$ and $\kappa_{2}^{\mathcal{E}}$ take on minimum values

$$
\kappa_{1, \text { min }}^{\mathcal{E}}=\frac{a}{c^{2}} \text { and } \kappa_{2, \text { min }}^{\mathcal{E}}=\frac{a}{b^{2}} .
$$

Furthermore, the minimum and maximum values of the ratio $\kappa_{\mathrm{r}}^{\mathcal{E}}=\kappa_{1}^{\mathcal{E}} / \kappa_{2}^{\mathcal{E}}$, a component of the measures defined in (3), are given by

$$
\kappa_{\mathrm{r}, \min }^{\mathcal{E}}=\frac{a^{2}}{c^{2}}, \kappa_{\mathrm{r}, \max }^{\mathcal{E}}=\max \left(\frac{a^{2}}{b^{2}}, \frac{b^{2}}{c^{2}}\right) .
$$

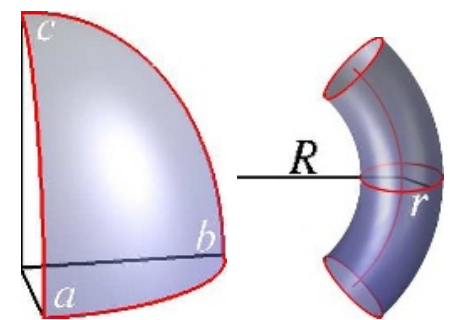

(a)

(b)

Fig. 3. The local shape of the isosurfaces of nodules is approximated by ellipsoidal patches (a) and that of vessels is approximated by toroidal patches (b)

Toroidal model. A torus $\mathcal{T}$ with small radius $r$ and large radius $R$, as shown in Fig. 3 b), can be parameterized as

$$
\mathcal{T}:\left\{\begin{array}{l}
x=(R+r \cos \psi) \cos \theta \\
y=(R+r \cos \psi) \sin \theta \\
z=r \sin \psi
\end{array}\right.
$$

with $(\theta, \psi) \in(-\pi, \pi]^{2}$. Its principal curvatures $\kappa_{1}^{\mathcal{T}}$ and $\kappa_{2}^{\mathcal{T}}$ take on values

$$
\kappa_{1}^{\mathcal{T}}=\frac{\cos \psi}{R+r \cos \psi} \text { and } \kappa_{2}^{\mathcal{T}}=\frac{1}{r}
$$

and the minimum and maximum values of the ratio $\kappa_{\mathrm{r}}^{\mathcal{T}}=\kappa_{1}^{\mathcal{T}} / \kappa_{2}^{\mathcal{T}}$ can be directly computed from (8) by varying $\psi$.

The analysis for the bounds in $\kappa_{1}, \kappa_{2}$, and $\kappa_{\mathrm{r}}$ presented in this section can be directly used to set an acceptable range for $\kappa_{1}$ and $\kappa_{2}$ and thresholds for (3). In the case of the ellipsoidal model, the range of values for $a, b$, and $c$ can be set based on the range of 
targeted nodule sizes. Given $a, b$, and $c$, we can set thresholds for $\kappa_{1 \text {,min }}^{\mathcal{E}}, \kappa_{2 \text {,min }}^{\mathcal{E}}$, and $\kappa_{\mathrm{r}, \min }^{\mathcal{E}}$. The lower bound on the aspect ratio for the ellipsoidal model was set at $1 / 2$, by assuming that nodules are roughly round. As for vessels, one typically has $R \gg r$, and thus $\kappa_{1, \text { min }}^{\mathcal{T}} \approx 0$. However, the surface of vessel junctions can be modeled as highly bending tori, which would result in $\kappa_{1, \operatorname{Tin}}^{\mathcal{T}} \ll 0$.

\section{Application to Lung Nodule Detection}

The algorithm was initially implemented using the open source Insight Toolkit (ITK) [14], which provides a general framework for processing $n$-dimensional medical images and includes basic libraries for image processing, segmentation and registration.

The volume image was first smoothed by convolution with a Gaussian kernel to reduce the effect of noise. Then the lung volume was automatically extracted via region growing to provide a region of interest (ROI) for subsequent operations. The eigenvalues of the curvature tensor were computed at every voxel in the ROI, and thresholds derived from the anatomical models were used to label each voxel as spherical, cylindrical, or neither.

A major source of false positives for the Hessian are vessel junctions, since they display large second derivatives in all directions. Junction isosurfaces, on the other hand, are clearly non-spherical, as seen in Fig. 4. However, they have sharp peaks that cause the curvature tensor to generate a thin streak of spurious spherical responses along the centerline of the vessels. These spurious responses can be easily removed by morphological closing of the surrounding cylindrical responses.

The responses defined at individual voxels are sufficient for presentation to radiologists as a tool for highlighting spherical regions, e. g., by overlaying the responses on the original CT volume, as shown in Fig. 5 To quantify the algorithm's performance neighboring voxel responses were grouped and counted as a single detection. Experimental results and validation are presented in

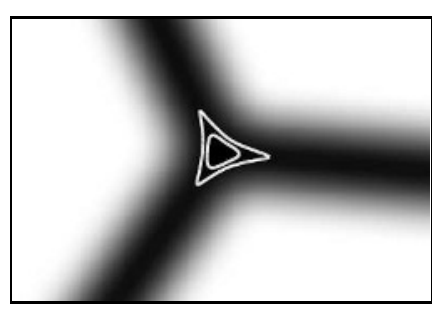

Fig. 4. Isosurfaces around the center of a junction display sharp peaks, with strong spherical responses along the centerline of the surrounding vessels

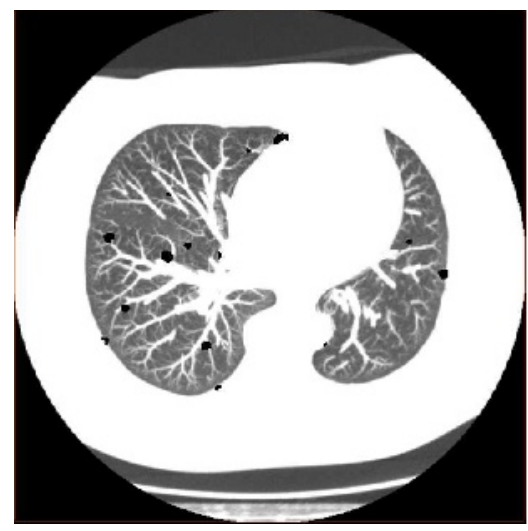

Fig. 5. A maximum intensity projection image showing spherical responses overlaid on the original CT data the next section.

The algorithm was re-implemented with several optimizations, including specialized data structures for memory access and morphological operations. On a 500-slice 


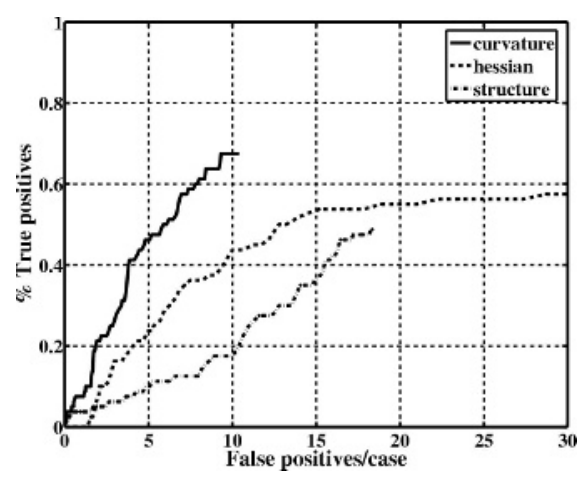

(a)

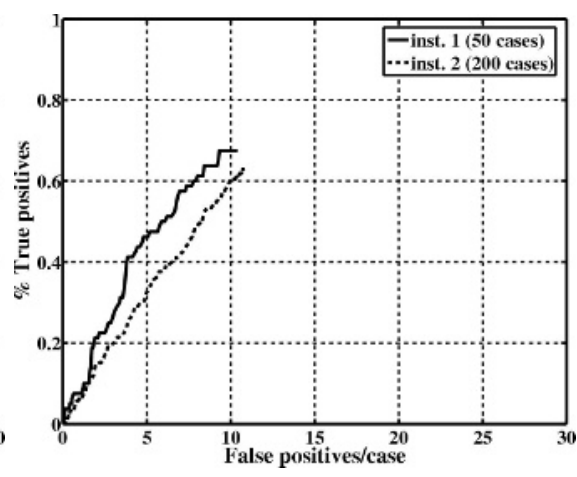

(b)

Fig. 6. (a) FROC curves showing the performance of the proposed algorithm compared to two other commonly-used methods. (b) FROC curves showing the algorithm's performance on datasets from two different institutions with different acquisition protocols.

dataset with slice thickness of $0.625 \mathrm{~mm}$, the optimized version ran in 10 seconds on off-the-shelf hardware with dual $3.06 \mathrm{GHz}$ Xeon processors and $2 \mathrm{~GB}$ of memory. The algorithm works with physical coordinates, using the pixel and slice spacing information contained in the CT images. For example, the standard deviation $\sigma$ of the smoothing kernel and the radii for the morphological structuring elements were specified in millimeters and selected based on the anatomical models and clinical protocol. The smoothing kernel has $\sigma=2 \mathrm{~mm}$, targeted at detecting nodules of diameter $4 \mathrm{~mm}$.

\section{Experimental Results}

The algorithm was validated on datasets from two different institutions, totaling 242 exams. The dataset from institution 1 consisted of 50 low-dose CT exams of high-risk subjects acquired at $40 \mathrm{mAs}$ and $120 \mathrm{kVp}$, with slice thickness of $1.25 \mathrm{~mm}$. The exams were independently read by three expert radiologists. Ground truth was defined as all nodules marked by two or more radiologists and consisted of 109 non-calcified solid nodules with diameter greater than $3 \mathrm{~mm}$ of which 80 had diameter greater than $4 \mathrm{~mm}$. The detections of the algorithm were considered true positives (TP) if they overlapped with a ground truth nodule, and false positives (FP) otherwise. Free response receiveroperator characteristic (FROC) curves were produced by sweeping the detections generated by the algorithm ranked in decreasing order of average spherical response.

For the nodules $4 \mathrm{~mm}$ and above in diameter, the algorithm achieved a sensitivity of $67.5 \%$ at $9.3 \mathrm{FP} /$ case. These results were compared with those of two other well-known and oft-used local shape descriptors: the Hessian [8] and the structure tensor [15]. At the same rate of $9.3 \mathrm{FP} /$ case, the Hessian and the structure tensor achieved sensitivities of only $40.0 \%$ and $17.5 \%$, respectively. The FROC curves for all three techniques are shown in Fig. 6(a), demonstrating the superior performance of the proposed technique. In another experiment, nodules with diameter between $3 \mathrm{~mm}$ and $4 \mathrm{~mm}$ were also included, since $3 \mathrm{~mm}$ is the smallest diameter for which a clinical protocol has 
been defined [7]. In this experiment, the proposed algorithm achieved a sensitivity of $70.6 \%$ at $25.6 \mathrm{FP} /$ case compared to sensitivities of $46.8 \%$ for the Hessian and $47.5 \%$ for the structure tensor at the same FP rate (FROC curve not shown).

The dataset from institution

Table 1. The table shows, for each radiologist, the number of nodules missed, the radiologist sensitivity, the number of missed nodules detected by the algorithm, the algorithm's false positive rate, the sensitivity improvement, the combined sensitivity of the radiologist and the algorithm, and the average sensitivity improvement weighted by the number of nodules on the ground truth for each radiologist. The difference in the algorithm's false positive rate is due to the different ground truth for each radiologist.

\begin{tabular}{|c|c|c|c|c|c|c|c|}
\hline & radiol & $\begin{array}{c}\text { missec } \\
\text { nod. }\end{array}$ & $\begin{array}{l}\text { initial } \\
\text { sensit. }\end{array}$ & $\begin{array}{l}\text { added } \\
\text { detect. }\end{array}$ & $\begin{array}{l}\text { FP/ } \\
\text { case }\end{array}$ & $\begin{array}{l}\text { sensit. } \\
\text { improv. }\end{array}$ & \begin{tabular}{|c|} 
final \\
sensit.
\end{tabular} \\
\hline & $\bar{A}$ & 6 & $86.4 \%$ & 3 & 10.0 & $6.8 \%$ & $93.2 \%$ \\
\hline$\forall$ & $\bar{B}$ & 6 & $86.4 \%$ & 4 & 10.0 & $9.1 \%$ & $95.4 \%$ \\
\hline & $\mathrm{C}$ & 30 & $55.9 \%$ & 19 & 9.5 & $27.9 \%$ & $83.8 \%$ \\
\hline & $\overline{\mathrm{D}}$ & $\overline{18}$ & $85.4 \%$ & $\overline{4}$ & 10.8 & $3.3 \%$ & $88.6 \%$ \\
\hline 5 & $\mathrm{E}$ & 19 & $84.7 \%$ & 16 & 10.8 & $12.9 \%$ & $97.6 \%$ \\
\hline$\cong$ & $\mathrm{F}$ & 68 & $60.7 \%$ & 36 & 10.5 & $20.8 \%$ & $81.5 \%$ \\
\hline \multicolumn{7}{|c|}{ avg. improv.: $14.2 \%$} & \\
\hline
\end{tabular}

2 consisted of 192 low-dose CT scans acquired using a different protocol (20 mAs, $140 \mathrm{kVp}$, with slice thickness of $2.5 \mathrm{~mm}$ ). Ground truth was defined as before and consisted of 210 noncalcified solid nodules with diameter above $4 \mathrm{~mm}$. The algorithm achieved a sensitivity of $62.9 \%$ at $10.3 \mathrm{FP} / \mathrm{case}$. As seen in Fig. 6(b), there is only a minor degradation in the algorithm's performance when compared to the results on the previous dataset, even though that was acquired at half the slice thickness.

To estimate the improvement in radiological sensitivity the algorithm's detections were combined with each radi-

ologist's reads. Using a leave-one-out scheme the ground truth for each radiologist was defined as only those nodules marked by the other two radiologists from the same institution. For institution 1 , there were 38 nodules that were marked by all three radiologists. For radiologist A, 6 nodules were considered "missed," i. e., marked only by B and $\mathrm{C}$. Therefore, the ground truth defined by radiologists $\mathrm{B}$ and $\mathrm{C}$ consisted of 44 nodules $(38+6), 38$ of which were marked by A, yielding an initial sensitivity of $86.4 \%$. The algorithm added three more detections, which, when combined with those of A, resulted in a final sensitivity of $93.2 \%$, at a cost of 10 false positives per case. This procedure was repeated for radiologists $\mathrm{B}$ and $\mathrm{C}$, and for the radiologists from institution 2 . The corresponding sensitivity improvements are shown in Table 1 . The overall average sensitivity improvement was $14.2 \%$. Figure 1 shows examples of nodules detected by the algorithm but not marked by one radiologist.

\section{Conclusions}

This paper introduces a technique for detecting pulmonary nodules in CT volume images of the lung that is based on combining geometric and intensity models with the eigenanalysis of the curvature tensor. The method does not require either segmentation of the relevant anatomical structures or sophisticated classifiers and can be viewed as a filter that highlights specific anatomical shapes. Furthermore, the algorithm does not 
require any training data as values of all parameters and thresholds are derived analytically from the models. The method is robust to changes in scanning protocols, including slice thickness, as demonstrated by the algorithm's performance on multiple databases with radiologist-provided ground truth.

\section{References}

1. Mulshine, J.L., Smith, R.A.: Lung cancer 2: Screening and early diagnosis of lung cancer. Thorax 57 (2002) 1071-1078

2. McCulloch, C.C., Yankelevitz, D., Henschke, C., Patel, S., Kazerooni, E., Sirohey, S.: Reader variability and computer aided detection of suspicious lesions in low-dose CT lung screening exams. Radiology 226 (2003) 37A

3. Brown, M.S., Goldin, J.G., Suh, R.D., McNitt-Gray, M.F., Sayre, J.W., Aberle, D.R.: Lung micronodules: Automated method for detection at thin-section CT - initial experience. Radiology 226 (2003) 256-262

4. McCulloch, C.C., Kaucic, R.A., Mendonça, P.R.S., Walter, D.J., Avila, R.S.: Model-based detection of lung nodules in computed tomography exams. Academic Radiology 11 (2004) 258-266

5. Paik, D.S., Beaulieu, C.F., Rubin, G.D., Acar, B., Jeffrey, Jr., R.B., Yee, J., Dey, J., Napel, S.: Surface normal overlap: A computer-aided detection algorithm with application to colonic polyps and lung nodules in helical CT. IEEE Trans. Medical Imaging 23 (2004) 661-675

6. Farag, A.A., El-Baz, A., Gimel'farb, G.G., Falk, R., Hushek, S.G.: Automatic detection and recognition of lung abnormalities in helical CT images using deformable templates. In Barllot, C., Haynor, D.R., Hellier, P., eds.: Medical Image Computing and Computer-Assisted Intervention. Number 3217 in Lecture Notes in Computer Science, Saint-Malo, France (2004) $856-864$

7. ELCAP: International early cancer action program - Protocol (2003) http://icscreen. med.cornell.edu/ ielcap.pdf.

8. Sato, Y., Westin, C., Bhalerao, A., Nakajima, S., Shiraga, N., Tamura, S., Kikinis, R.: Tissue classification based on 3D local intensity structures for volume rendering. IEEE Trans. Visualization and Computer Graphics. 6 (2000) 160-180

9. Yoshida, H., Näppi, J.: Three-dimensional computer-aided diagnosis scheme for detection of colonic polyps. IEEE Trans. Medical Imaging 20 (2001) 1261-1274

10. Vos, F.M., Serlie, I.W.O., van Gelder, R.E., Post, F.H., Truyen, R., Gerritsen, F.A., Stoker, J., Vossepoel, A.M.: A new visualization method for virtual colonoscopy. In Niessen, W.J., Viergever, M.A., eds.: Medical Image Computing and Computer-Assisted Intervention. Number 2208 in Lecture Notes in Computer Science, Berlin, Springer-Verlag (2001) 645-654

11. O’Neill, B.: Elementary Differential Geometry. Academic Press, New York (1966)

12. Forsyth, D.A.: Shape from texture and integrability. In: Proc. 8th Int. Conf. on Computer Vision. Volume II., Vancouver, Canada (2001) 447-452

13. Yankelevitz, D.F., Reeves, A.P., Kostis, W.J., Zhao, B., Henschke, C.I.: Small pulmonary nodules: Volumetrically determined growth rates based on CT evaluation. Radiology 217 (2000) 251-256

14. Ibáñez, L., Schroeder, W., Ng, L., Cates, J.: The ITK Software Guide. Kitware Inc. (2003)

15. Krissian, K., Malandain, G., Ayache, N., Vaillant, R., Trousset, Y.: Model based detection of tubular structures in 3D images. Computer Vision and Image Understanding 80 (2000) $130-171$ 